behaviour (e.g. driving, active travel, public transit, walking, and cycling) from adults in the general population. Interventions were categorized into functions (how the intervention seeks to affect behaviour). Depending on whether gains or losses of functions could occur, interventions were classified as carrots (e.g. new bikeshare programs), combined carrot and stick (e.g. traffic calming), or stick interventions (e.g. congestion charging). Harvest plots were used to visually summarize the findings weighted by study quality. Where possible, outcomes were converted into standardized mean differences (SMD) and random-effects meta-analyses were conducted.

Results We extracted data from 83 publications reporting 98 interventions. From these, we identified 20 intervention types and eight function categories. The majority of interventions were carrots $(n=64)$, followed by carrot and stick $(n=17)$ and stick $(n=17)$. Harvest plots demonstrated that most evaluations, particularly those classified as higher quality, found changes in favour of the intervention. Results for carrot interventions, however, were more less consistent than for stick or combined interventions. This was consistent with findings from the meta-analysis, which were statistically nonsignificant but had point-estimates of greater magnitude for driving outcomes for sticks (SMD -0.21; 95\%CI -0.43, 0.01) and combined carrot and stick interventions $(-0.17 ;-0.65,0.31)$ compared to carrots $(-0.09 ;-0.21,0.03)$. Likewise, for active travel outcomes, combined carrot and stick interventions had a higher SMD $(0.39 ;-0.01,0.78)$ compared to carrot interventions $(0.10 ;-0.06,0.25)$. Financial functions were found to be the most effective for driving outcomes, whereas access, convenience, safety, and space were more effective for active travel outcomes.

Discussion This is the first review to compare whether positive, negative, or combined strategies and their functions differ in terms of effectiveness on travel behaviour, which can aid policymakers in designing sustainable transportation policies. Further research is needed for interventions with a stick component, which suggest greater effectiveness yet remain less well-studied, possibly because they are less conducive to experimental manipulation.

\section{OP51 ACTIVE GROUP-BASED PERFORMING ARTS INTERVENTIONS FOR PARKINSON'S DISEASE: SYSTEMATIC REVIEW AND META-ANALYSIS}

${ }^{1}$ Maxwell Barnish*, ${ }^{2}$ Susannah Barran. 'Peninsula Technology Assessment Group (PenTAG), University of Exeter, Exeter, UK; ${ }^{2}$ Children and Young People's Speech and Language Therapy, Guy's and St Thomas' NHS Foundation Trust, London, UK

\subsection{6/jech-2021-SSMabstracts.51}

Background Parkinson's disease (PD) is a common age-related neurodegenerative condition associated with a wide range of motor and non-motor symptoms. There has been increasing interest in performing arts interventions for $\mathrm{PD}$, due to the limitations of pharmacological therapies. As no prior systematic review had addressed a wide range of artistic modalities, we systematically evaluated the potential benefit of all active group-based performing arts interventions in PD.

Methods In order to systematically evaluate the benefit of performing arts interventions in $\mathrm{PD}$, searches on PsycINFO, AMED, CINAHL, EMBASE, and MEDLINE were performed in February 2020. Supplementary web searches and citation chasing were conducted. Included studies used any quantitative design to assess the benefit of any active group-based performing arts intervention on quality of life, functional communication, speech, motor function or cognitive status for people with diagnosed PD. Following standardised duplicate screening and data extraction, data synthesis was conducted using thematic narrative synthesis and random effects metaanalyses using Review Manager 5.3. Risk of bias was evaluated using SURE checklists (Cardiff University).

Results Searches identified 2669 records, of which 260 proceeded to initial full-text review following de-duplication, 129 to detailed full-text review and 67 to inclusion in the systematic review, representing 56 separate studies. Data from 10 separate studies were included in meta-analyses. Risk of bias assessment revealed limitations across many studies. Dance was the most studied artistic modality (39 studies), followed by singing (12 studies), music therapy (4 studies) and theatre (2 studies). Narrative synthesis showed evidence for a benefit of dance, music therapy and singing on quality of life; singing on speech; dance and music therapy on cognition; and dance, music therapy, singing and theatre on motor function. There was no evidence of benefit on functional communication. In meta-analyses, tango-based dance predicted superior UPDRS motor than usual care $(\mathrm{Z}=2.87, \mathrm{p}=0.004)$ and superior timed-up-and-go than exercise $(Z=3.77, p=0.0002)$, while PD-specific dance predicted superior PDQ-39 quality of life than usual care $(Z=3.77, p=0.0002)$.

Conclusion Performing arts interventions may offer benefit in PD. However, it is difficult to assess which performing arts modalities may be most beneficial for which outcomes, due to a lack of studies directly comparing different performing arts interventions. Key limitations included small sample sizes, differences in comparator interventions, differences in intervention frequency and duration, as well as differing disciplinary backgrounds of session leaders. Further research is needed with greater methodological rigour before firm conclusions can be drawn.

\section{OP52 IDENTIFYING LOCAL AUTHORITY NEED FOR, AND UPTAKE OF, SCHOOL-BASED PHYSICAL ACTIVITY INTERVENTIONS IN ENGLAND - A CLUSTER ANALYSIS USING ROUTINE DATA}

${ }^{1}$ Tishya Venkatraman*, ${ }^{1}$ Kate Honeyford, ${ }^{1}$ Bina Ram, ${ }^{2}$ Esther MF van Sluijs, ${ }^{1}$ Céire E Costelloe, 'Sonia Saxena. '1Department of Primary Care and Public Health, Imperial College London, London, UK; ${ }^{2} 2 M R C$ Epidemiology Unit and Centre for Diet and Activity Research (CEDAR), University of Cambridge, Cambridge, UK

\subsection{6/jech-2021-SSMabstracts.52}

Background Schools in England need effective, simple interventions to address shortfalls in children's physical activity. Areas in England where physical activity is low among children may particularly benefit from school-based physical activity interventions. Further, stark geographical inequalities for children's physical activity exist and have widened during Covid-19 lockdowns. The Daily Mile (TDM) is a school-based running programme that has been widely adopted across England. However, there is limited evidence of its equitability and whether it reaches children in most need. We aimed to i) identify local authorities in England with the highest need for children's physical activity promotion and ii) determine whether TDM reaches schools in areas of high need.

Methods This was a cross-sectional study linking routinely collected data from a range of sources including the Active Lives Surveys and national datasets. Characteristics of need were 